\title{
EL DICCIONARIO CONTRASTIVO PORTUGUÉS- ESPAÑOL (DICOPOES) EN LA LEXICOGRAFÍA BILINGÜE PORTUGUÉS-ESPAÑOL: APORTACIONES, LIMITACIONES Y EXPECTATIVAS
}

M. ${ }^{a}$ Ángeles Sastre Ruano

Universidad de Valladolid

sastre@fyl.uva.es

Resumen: En este trabajo se describen algunos aspectos del Diccionario Contrastivo Portugués-Español (DiCoPoEs), un diccionario concebido como un diccionario de aprendizaje de español dirigido a individuos que tienen el portugués como lengua materna $\mathrm{y}$, más exactamente, la variante brasileña del portugués.El equipo que está elaborando el DiCoPoEs ha apostado por el diccionario bilingüe contrastivo para el aprendizaje de lenguas extranjeras y pretende demostrar cómo puede combinarse la instrucción en el uso de una lengua extranjera (el español) por medio de los llamados equivalentes del diccionario bilingüe con información contrastiva relativa a las diferencias semánticas entre las unidades de la lengua materna (el portugués) que figuran como punto de partida en los artículos lexicográficos (los lemas) y las voces que se presentan como correspondientes en la lengua de destino (los equivalentes).

Palabras clave: lexicografía bilingüe contrastiva, diccionario contrastivo, diccionario de aprendizaje, portugués-español, información contrastiva, equivalentes

CONTRASTIVE DICTIONARY OF PORTUGUESE AND SPANISH (DICOPOES) IN THE BILINGUAL LEXICOGRAPHY OF PORTUGUESE AND SPANISH: CONTRIBUTIONS, LIMITATIONS AND EXPECTATIONS 


\begin{abstract}
Some aspects of the Contrastive Portuguese and Spanish Dictionary (DiCoPoEs) are described in this work. This dictionary has been created as a learner's dictionary of Spanish, geared for individuals who have Portuguese as their native language, specifically, Brazilian Portuguese. The team writing the DiCoPoEs supports the contrastive bilingual dictionary for the learning of foreign languages. Their aim is to demonstrate how instruction in the use of a foreign language (Spanish) through the use of named equivalents of the bilingual dictionary can be combined with contrastive information which is relative to the semantic differences among the words of the native language (Portuguese). These semantic differences represent a point of departure in the lexicographic articles (the headwords) and the voices that are presented as correspondents in the target language (equivalents)

Key words: Contrastive bilingual lexicography, contrastive dictionary, learner's dictionary, Portuguese-Spanish, contrastive information, equivalents
\end{abstract}

La elaboración de un Diccionario Contrastivo Portugués-Español (DiCoPoEs) es la meta de un proyecto realizado entre profesores investigadores de tres universidades: la Universidade Federal de Santa Catarina, la Universidad de Augsburgo y la Universidad de Valladolid.

El diccionario está concebido como un diccionario de aprendizaje de español dirigido a individuos que tienen el portugués como lengua materna y, más exactamente, la variante brasileña del portugués.

La justificación metodológica que subyacen en este diccionario ya fue apuntada en un trabajo previo presentado en el marco del IV Congreso Internacional de Lexicografía Hispánica, celebrado en Tarragona en septiembre de 2010 (Balbino de Amorim Barbieri Durão, Sastre Ruano y Werner, 2012). Pero no está de más recordarla:

El diccionario bilingüe puede, desde varias perspectivas, prestar mejores servicios como diccionario de aprendizaje que el diccionario monolingüe. 
El diccionario bilingüe, especialmente cuando va dirigido a estudiantes de lengua extranjera, no debe ser concebido como un mero «diccionario de equivalentes» 0 «diccionario de equivalencias», sino como diccionario contrastivo.

La contrastividad del diccionario bilingüe debe concebirse en dependencia del grado de parentesco o de diferencias entre las dos lenguas de la obra lexicográfica.

Dado que estas justificaciones distan de tener una aceptación unámine, conviene que estén justificadas.

La mayoría de los diccionarios de aprendizaje modernos son diccionarios monolingües. Muchos expertos en didáctica de idiomas extranjeros y muchos lingüistas especializados en lexicografía sostienen que los diccionarios bilingües, por su naturaleza, no pueden ser buenos diccionarios para el aprendizaje de lenguas extranjeras.

Los argumentos en contra del diccionario bilingüe como diccionario didáctico atañen a los siguientes aspectos: a) los que destacan las diferencias semánticas entre las lenguas; b) la imposibilidad de delimitar los significados léxicos de una lengua por medio de la indicación de los llamados equivalentes en otra lengua; y c) el peligro de la interferencia que se piensa debe resultar de la igualación consiguiente entre significados de una y otra lengua por parte del aprendiz de la lengua de destino del diccionario. Se piensa que el significado de una unidad léxica de una lengua solo puede ser explicado mediante el recurso a otros elementos de la misma lengua y que no puede ser explicado satisfactoriamente mediante equivalentes por traducción. De esta última observación se deduce que solo el diccionario monolingüe sería capaz de explicar los significados léxicos, mientras que el bilingüe podría explicarlos solo de manera insuficiente y, en el contexto de la enseñanza de 
una lengua no materna, induciría a interferencias indeseables. El peligro de la interferencia semántica pesaría aún más en el caso de lenguas con vocabularios de extrema afinidad, como es el caso del portugués y el español.

Nuestro equipo ha apostado por el diccionario bilingüe contrastivo para el aprendizaje de lenguas extranjeras y ha demostrado cómo puede combinarse la instrucción en el uso de una lengua extranjera (el español) por medio de los llamados equivalentes del diccionario bilingüe con información contrastiva relativa a las diferencias semánticas entre las unidades de la lengua materna (el portugués) que figuran como punto de partida en los artículos lexicográficos (los lemas) y las voces que se presentan como correspondientes en la lengua de destino (los equivalentes).

El diccionario bilingüe alfabético es el diccionario onomasiológico más cómodo para el aprendiente de un idioma extranjero. Como obra de consulta orientada a la producción textual y discursiva, a un hablante de portugués le resulta mucho más cómodo y rentable buscar la expresión española que expresa el concepto de beca con la acepción de 'ayuda económica que se concede a un estudiante para que complete sus estudios o para que realice una investigación determinada' partiendo de la palabra bolsa en un diccionario portugués-español, que encontrar la palabra beca e identificarla como la palabra adecuada con ayuda de un diccionario onomasiológico español monolingüe.

Uno de los argumentos que parecen abogar en contra del uso del diccionario bilingüe en el contexto didáctico es el peligro de que este favorezca las interferencias de la lengua que es el punto de partida en la búsqueda de expresiones. Para paliar esto, es decir, para remediar estas interferencias que pudieran surgir como consecuencia de la consulta de un diccionario bilingüe, surgen las notas explícitamente contrastivas, orientadas a prevenir mediante explicaciones la posible interferencia. Las explicaciones léxicas y semánticas contrastivas no puede ofrecerlas un diccionario monolingüe.

Veamos los siguientes ejemplos: 
cachaça $s f 1$ cachaza $s f$, aguardiente de caña $s m$, aguardiente de melaza sm NOTA Em espanhol, também se utiliza a palavra cachaza (ou seus equivalentes parsimonia, flema ou pachorra) para referir-se à tranquilidade excessiva que se sente ao desenvolver uma atividade.

[Sobra decir aquí que es una bebida alcohólica muy popular en Brasil, que se obtiene por la fermentación y posterior destilación del caldo de la caña de azúcar, que tiene elevada graduación alcohólica y que los brasileños se refieren a ella con otros nombres más populares como pinga, branquinha o mel. ¿Y por qué sobra esta información? Porque se presupone que los hablantes de portugués ya la conocen.]

corrida $s f$ (ato de correr; de taxi; disputa esportiva) carrera $s f$ NOTA Em espanhol, a palavra corrida (o corrida de toros) também designa um espetáculo público no qual touros bravos são desafiados em uma arena fechada ou plaza de toros e na qual, normalmente, participam três matadores ou toreros, cada um dos quais luta com dois touros bravos. NOTA Em espanhol, a palavra carrera serve para fazer referência ao conjunto de estudos universitários que habilitam para o exercício de uma profissão.

Estos son argumentos que atañen solo a los diccionarios de aprendizaje concebidos como diccionarios de producción. Pero un diccionario de aprendizaje puede, además, ser otro tipo de diccionario, concebido no solo como herramienta que sirva como obra de consulta en casos de duda puntual, sino también como obra con 
la ayuda de la cual se pueda aprender, sistemáticamente, el uso de unidades léxicas de una lengua. Un diccionario de apendizaje puede ser algo más que un diccionario al uso de aprendices de la lengua (un learner's dictionary): puede ser también una obra con la ayuda de la cual se adquiere, con cierta sistematicidad, vocabulario de una lengua (un learning dictionary). En este último sentido, un diccionario portugués-español podría ser consultado no solo en casos de duda puntual acerca de una determinada característica del uso de una palabra, sino también para informarse, de una vez por todas, del total de las diferencias entre un número de voces de las dos lenguas que mantienen entre sí una relación de heteronimia más o menos completa.

calçada $s f$ acera $s f$ NOTA Em espanhol, a acera é a parte pavimentada da rua que está destinada aos transeúntes, por isso é um pouco mais alta que o restante da rua; já, calzada é a parte da rua compreendida entre duas calçadas, parte esta que está destinada ao trânsito de carros e demais veículos motorizados ou não.

camada $s f \mathbf{1}$ (quantidade de matéria que se estende mais ou menos uniformemente sobre uma superfície) capa $s f$, mano $s f \mathbf{2}$ (faixa de certa substância entreposta ou sobreposta a outra) capa $s f 3$ (estrato, classe ou categoria social) clase $s f$ NOTA Em espanhol, o substantivo feminino camada é usado para fazer referência, por um lado, ao conjunto dos filhotes de um mamífero nascidas de uma vez; por outro, a um conjunto de pessoas, geralmente de má reputação (como malandros, ladrões e criminosos), as que alguém se refere com desprezo. 


\begin{abstract}
caminhada $s f$ (jornada ou passeio que se faz a pé) paseo $s m$, caminata $s f$ NOTA Em espanhol, emprega-se o termo paseo para fazer referência a uma distância curta que pode ser percorrida em pouco tempo e também a um percurso feito a pé ou de outro modo para passear ou com um objetivo que não seja chegar a um lugar específico; o termo caminata, por outro lado, indica um percurso longo e cansativo feito a pé.
\end{abstract}

Para que un diccionario bilingüe supere al monolingüe en funcionalidad, es necesario que se reformule el papel que desempeña en él la indicación de equivalentes. El papel central en la información que proporciona el diccionario no lo debe jugar la presentación de equivalentes a la que, más o menos sistemáticamente, se agregan algunas glosas diferenciadoras, sino que lo debe jugar la información abundante y sistemática acerca de las diferencias entre las unidades léxicas de una lengua y aquellas que suelen denominarse equivalentes, aunque sabemos que nunca son equivalentes en sentido estricto.

El diccionario bilingüe, especialmente el de aprendizaje, debe concebirse no como diccionario de equivalencias o de equivalentes, sino como un diccionario contrastivo. El diccionario bilingüe debe presentar los tradicionales equivalentes de tal manera que el usuario se dé cuenta de que no siempre son equivalentes y que se entere de cuáles son las diferencias de uso entre las voces de partida del diccionario y los equivalentes no propiamente equivalentes de la lengua de destino (la lengua objeto de aprendizaje). Sostenemos que la información contrastiva en la indicación de equivalentes ha de ser el contenido central de los artículos de un diccionario bilingüe de producción y aprendizaje. prado $s m$, pradera $s f$ NOTA Em espanhol, a diferença 
entre um prado e uma pradera é o fato de a pradera ser maior que um prado. 2 (planicie) llanura $s f$ NOTA Em espanhol, a palavra campiña é empregada para fazer referência ao campo pela beleza que atrai os sentidos.

Una vez concebido el diccionario bilingüe como diccionario contrastivo, resulta obvio que pueden variar, según el grado de afinidad léxica y gramatical entre las dos lenguas del diccionario, las proporciones entre los dos componentes funcionales, la de aprovechar posibilidades de transferencia deseable y la de prevenir la transferencia indeseable. Las posibilidades de transferencia se dan especialmente en casos de estrecha afinidad entre dos lenguas (cual es el caso de las lenguas que nos ocupan); sin embargo, un alto grado de afinidad entre las dos lenguas fomenta también las interferencias. Esto significa que en diccionarios de pares de lenguas como portugués-español podría ahorrarse mucho espacio suprimiendo información redundante $\mathrm{y}$, a la vez, aprovechar el espacio ahorrado para desarrollar la información contrastiva y concentrarse más en las diferencias de uso que en las características que comparte una voz de una lengua con una de otra lengua.

canastra $s f$ canasta $s f$, canasto $s m$ NOTA Em espanhol, a palavra canasto designa um recipiente de tamanho maior que o designado pela palavra canasta.

carro $s m 1$ (veículo) coche $s m 2$ (vagão) coche $s m$, vagón $s m 3$ (de boi) carreta $s f$, carro $s m$ nоTA Em espanhol, a diferença entre carro e carreta está no fato de que carreta é designa um veículo de forma mais alargada e estreita que o carro. 4 (de máquina de escrever) carro $\mathrm{sm}$ 
casaco $s m 1$ chaqueta $s f$, americana $s f$, cazadora $s f$ NOTA Em espanhol, a chaqueta pode ser de lã ou de tecido e se caracteriza por ter uma abertura na frente e ser ajustada ao corpo; a americana tem solapa e é abotoada com botões, além de costumar ter bolsos na frente e poder ser cruzada ou sem cruzar; a cazadora se ajusta à cintura, normalmente com um elástico ou com uma tira que se amarra. 2 abrigo sm nOTA Em espanhol, a palavra abrigo designa uma roupa mais formal usada no inverno por cima da roupa para sair à rua.

Ahora bien, este equipo de investigadores tiene plena conciencia de que no ha sido el primero en esbozar un plan para la confección de un diccionario didáctico bilingüe. No obstante, tengo que destacar aquí algunas diferencias sustanciales entre nuestro proyecto y otros que, a primera vista, podrían parecer del mismo tipo. Últimamente ha aumentado bastante el número de los diccionarios que nosotros llamamos bilingualizados (que no son otra cosa que diccionarios originariamente monolingües en los que una parte de la información sobre la lengua objeto del diccionario, que para el estudiante es la lengua que aprende y que en el diccionario monolingüe es también la lengua en la que se ofrece la información, es sustituida o ampliada por información proporcionada en la lengua materna del usuario. La información que se brinda en la lengua materna del usuario puede abarcar, por ejemplo, traducciones de las explicaciones semánticas o la presentación de equivalentes en la lengua materna del usuario, que pueden acompañar estas explicaciones, así como traducciones de diversos tipos de indicaciones y acotaciones, como aquellas que en español se denominan marcas (gramaticales, estilísticas, etc.)

En nuestro caso, el DiCoPoEs aprovecha el mecanismo de transferencia positiva de conocimientos de la lengua materna (el 
portugués) hacia la segunda lengua que se aprende (el español) porque lleva al usuario desde voces de su lengua materna hacia voces de la lengua extranjera que necesita para producir un texto o discurso en la lengua que están aprendiendo.

\section{$\square_{\text {¿Cómo hemos aplicado la contrastividad? }}$}

La función del DiCoPoEs no es explicar unidades léxicas de la lengua de partida del diccionario (para esto están los diccionario monolingües, que estructuran las acepciones de acuerdo con criterios determinados en cada caso; e incluso los bilingualizados). Pero sí nos aprovechamos de ellas como punto de arranque en la búsqueda de unidades léxicas equivalentes en la lengua de destino. Obviamente, debido a la anisomorfía léxico-semántica entre la lengua de partida y la de destino, con frecuencia a una voz de la lengua de partida le corresponden varios equivalentes en la lengua de destino.

Cuando esto sucede (sucede muchas veces, claro está), es decir, cuando se ofrecen varios equivalentes españoles para una unidad léxica del portugués, hay que explicar las condiciones que determinan el uso de cada una de las voces españolas ofrecidas. En algunos casos estás condiciones serán de índole semántica; en otros, sintáctica; en otros, pragmática.

chinelo $s m$ chinela $s f(!)$, chancleta $s f$, chancla $s f$, zapatilla, $s f$ NOTA Em espanhol, todos esses termos têm em comum a referência a um calçado sem cobertura para o calcanhar que normalmente é usado dentro de casa; a palavra zapatilla faz referência também a um calçado que tem cobertura no calcanhar e saltinho; as palavras chancla e chancleta também fazem referência a um calçado que pode ser usado fora de casa. 
Cuando no aparece ninguna explicación de este tipo, entendemos que los equivalentes propuestos pueden funcionar como sinónimos en todos los contextos (sinónimos funcionales).

cravo sm 1 (planta; prego) clavo sm 2 (flor) clavel sm 3 (instrumento) clavicémbalo $s m$, clave $s m$

cremação $s f \underline{\text { incineración }} s f$, cremación $s f$

criado, -a I adj criado, -a adj II $s m / f$ (pessoa que presta serviços domésticos) criado, -a $s m / f$, sirviente, -a $s m / f$

curtume $s m 1$ (curtimento de couro) curtido sm, curtición $s f$, curtimiento $s m 2$ (estabelecimento onde se curte couro) curtiduría $s f$, tenería $s f$

Para ayudarnos en el caso de diferencias semánticas (que suelen ser las más frecuentes) entre las voces españolas presentadas como equivalentes, las indicaciones diferenciadoras se formulan como explicaciones explícitas en forma de paráfrasis o en alguna otra forma que contextualiza la voz española (glosa), obviamente con la acepción que le corresponde a la voz portuguesa que figura como lema.

chave $s f \mathbf{1}$ (utensílio para fechar e abrir; dispositivo para conectar-se à rede elétrica) llave $s f \mathbf{2}$ (em escritura musical; código; elemento que proporciona a compreensão de alguma idéia) clave $s f$

charlatão sm (mercador ambulante que vende drogas e elixires reputados milagrosos, atraindo e iludindo 
o público) charlatán sm NOTA Em espanhol, charlatán, - $a$, como adjetivo, é usado para fazer referência a uma pessoa que fala muito sobre assuntos sem a menor relevância.

\section{certo, -a adj 1 (verdadeiro, baseado em fatos reais) cierto adj 2 (correto) correcto, -a adj 3 (adecuado) adecuado, -a adj 4 (garantido, -a) asegurado, -a adj, garantizado, -a adj 5 (determinado) fijado, -a adj}

cenário $s m 1$ (palco) escenario $s m 2$ (no teatro) decorado $s m 3$ (escándalo) número $s m$, numerito $s m$, escena $s f$

Las indicaciones semánticas que anteceden a los equivalentes no deben interpretarse como explicaciones de las acepciones de la palabra portuguesa (insistimos: para eso están los diccionarios monolingües), sino como información sobre las intersecciones entre el significado de la palabra portuguesa y los significados de la palabra española que puede servir para referirse a los conceptos a los que el usuario lusohablante del diccionario quiere referirse en español. Su función es la de suministrar la información contrastiva acerca de las diferencias semánticas entre las dos lenguas, aunque contrastándolas solo en una dirección: la del portugués al español (la otra dirección, por ahora, la tenemos aparcada en la recámara y mantenemos la esperanza de que no pase al baúl de los recuerdos).

Donde prevemos que puede existir peligro de que usuario del diccionario llegue a conclusiones erróneas a través de la información ofrecida, se agregan observaciones contrastivas en dirección inversa. de um animal) dentadura $s f$ NOTA Em espanhol, não é 
suficiente usar dentadura para fazer referência à dentatura postuça. É preciso dizer dentadura artificial o dentadura postiza, já que dentatura significa, nessa língua, dentição.

descartável adj (que se utiliza uma única vez) desechable adj NOTA Em espanhol, o adjetivo descartable se aplica a uma possibilidade, ideia ou circunstância que pode ser recusada ou que oide ser prescindida por ser considerada pouco conveniente ou pouco plausivel.

chouriço $s m$ morcilla $s f$ NOTA Em espanhol, a palavra chorizo, com a acepção de 'um tipo de frio', equivalendo, ao português, à palavra linguiça.

Los problemas se perciben con más claridad cuando se contrastan palabras portuguesas con palabras etimológicamente emparentadas del español, de forma ortográfica igual o muy parecida, pero con acepciones asimétricamente distribuidas:

camarote $s m 1$ (em um teatro) palco $s m 2$ (em navio) camarote $\mathrm{sm}$

carrinho $s m 1$ (de bebê; de supermercado) carrito $s m$ 2 (de parque de diversões) coche de choque $s m$

carteira $s f 1$ (de dinheiro) billetera $s f$, cartera $s f 2$ (de ações, de documentos) cartera $s f \mathbf{3}$ (pacote de cigarros) cajetilla sf 4 (escrivaninha) escritorio $s m 5$ (de sala de aula) pupitre $s m 6$ (documento de identidade) carné $s m$, carnet $s m$ 
abono sm 1 (dinheiro pago) abono sm, pago sm 2 (pago antecipadamente) adelanto $s m$, anticipo $s m 3$ (bônus) gratificación $s f$, paga extraordinaria $s f$, paga extra $s f$, aguinaldo $s m$ NOTA Em espanhol, esses equivalentes têm em comum o caráter adicional, acrescentado ou complementar, mas existem diferenças entre eles: uma gratificación pode ser em dinheiro ou em algum tipo de objeto com qualquer motivo; o que se chama paga extraordinaria (ou paga extra) é uma retribuição em dinheiro que um trabalhador recebe, geralmente duas vezes por ano, além do seu salário; o aguinaldo é uma espécie de presente ou gratificação dada a um trabalhador por ocasião do natal. 4 (de opinião) refuerzo argumentativo $s m$, corroboración $s f \mathbf{5}$ (fiança) aval $s m$, fianza $s f$, garantía $s f \mathbf{6}$ (de falta no trabalho) justificación $s f \mathbf{7}$ (aprovação) aprobación $s f \mathbf{8}$ (elogio) alabanza $s f$, elogio $s m$ NOTA Em espanhol, a palavra abono, além de significar pagamento de alguma dívida que se deve, significa, tambèm, a substância (orgânica ou inorgânica) que se introduz na terra para que ela se torne mais produtiva. Com este significado, esta palavra equivale à palavra portuguesa adubo. Também faz referência ao lote de entradas ou bilhetes que se compram ao mesmo tempo e que permitem a una pessoa o uso periódico de um serviço ou a assistência de uma série predeterminada de espetáculos.

También son frecuentes en este par de lenguas los casos en los que existen dos palabras de ortografía igual o muy parecida y, además, con étimo idéntico, pero sin cruce en ninguna de sus acepciones.

cobra I adj $\mathbf{1}$ (especialista em algo) experto, -a adj II $s f 2$ (réptil) culebra $s f$ NOTA Em espanhol, a pala- 
vra designa uma serpente muito venenosa, conhecida também como serpiente de anteojos, característica da África e do sul da Ásia. Essa serpente se caracteriza por ter um par de colmillos que servem para que injete veneno e por uma expansão da pele dos dois lados do pescoço, que lhe serve como mecanismo de defesa ou para assustar o inimigo. 3 (pessoa de má índole) víbora $s f$

Más problemas que complican la correlación entre acepciones y equivalentes: acepciones que un sustantivo tiene solo en plural o un verbo solo en forma pronominal, así como unidades pluriverbales, usos fraseológicos.

cadeira I sf $\mathbf{1}$ (disciplina ministrada em centro docente) asignatura $s f 2$ (lugar destinado a um político na câmara) escaño $s m$ II $s f p l 3$ (parte do corpo) cadera $s f$, caderas sfpl NOTA En espanhol, se usa a forma em singular para fazer referência a cada uma de duas partes que se destacam dos dois lados do corpo pelos ossos superiores da pelvis. Para mencionar o conjunto dessas duas partes, pode-se optar por usar tanto o singular como o plural.

caça I $s f$ (ação de caçar) caza $s f$ II $s m$ (avião) caza $s m$, avión de caza $s m$

Una vez presentadas las principales aportaciones, pasamos al aparatado de las limitaciones.

Dado el amplio número de vocablos que supondría analizar contrastivamente, el equipo se inclinó por realizar una selección de 
estos y acotar solo los llamados falsos amigos, entendiendo como tales no solo los heterosemánticos, sino también aquellos que desde diversos puntos de vista (ortográfico, gramatical, etc.) pueden jugarle una mala pasada al hablante de portugués como lengua materna en su aprendizaje de español. Así, el DiCoPoEs pasó a ser concebido como un macroproyecto formado por varios diccionarios. El que ahora nos ocupa es el DiFaPE (Diccionario de falsos amigos portugués-español).

¿Qué vocablos se incluyen en este diccionario teniendo en cuenta esta consideración 'ancha', por llamarla de algún modo, de los llamados falsos amigos?

(i) Aquellos en los que la sílaba tónica es distinta en español:

cabala $s f$ cábala $s f$

cavalaria $s f$ caballería $s f$

cérebro $s m$ cerebro $s m$

(ii) Aquellos que poseyendo gran semejanza gráfica se diferencian en algunas grafías:

cabisbaixo, -a adj cabizbajo, -a adj

caçarola $s f$ cacerola $s f$

cafezal $s m$ cafetal $s m$

caixa I $s f$ (recipiente) caja $s f$ II $s m / f$ (pessoa que cobra valores em una instituição comercial) cajero, -a $s m / f$

campeão, -ã I adj campeón, -ona $\operatorname{adj}$ II $s m / f$ campeón, -ona $s m / f$

(iii) Aquellos en los que, a pesar de la gran similitud gráfica, el género gramatical del término equivalente es distinto

camuflagem $s f$ camuflaje $s m(!)$

cárcere $s m 1$ (prisão) cárcel $s f(!)$, prisión $s f 2$ (prisão subterrânea) calabozo $\mathrm{sm}$ 
carrapato $s m 1$ (ácaro) garrapata $s f($ !) 2 (pessoa inoportuna) pesado, -a $s m / f$, plasta $s m / f$

chantagem $s f$ chantaje $s m(!)$

chupeta $s f \mathbf{1}$ (para tranquilizar os bebês) chupete $s m$ (!) $\mathbf{2}$ (para carregar bateria de carro) pinzas $s f p l$

cor $s f$ color $\operatorname{sm}(!)$

cume sm (parte mais alta de uma montanha ou elevação de terreno; ponto ou momento de máximo desenvolvimento, esplendor ou êxito de uma coisa ou pessoa) cumbre $s f(!)$

(iv) Aquellos que presentan regímenes sintácticos distintos:

credor, -a I adj (que é digno ou merecedor de algo) acreedor, -a $a d j$ NOTA Com esta acepção, aparece acompanhado das preposições $a$ ou $d e$. II $s m / f$ (pessoa ou entidade a que se deve dinheiro) acreedor, -a $s m / f$

Una limitación más, es de ley decirlo, tiene que ver con los compuestos pluriverbales. Entrarían en este grupo tanto los sustantivos y adjetivos separados por un guion (del tipo ferro-velho, pronto-socorroi o palavra-chave) como los sustantivos formados por dos palabras o por $\mathrm{N}$ de $\mathrm{N}$ (del tipo bicho do mato, carteira de identidade, chá de bebé, briga de galo, fim de semana, freio de mão, o folha de pagamento) o por $\mathrm{N}+$ Adj (del tipo frase feita o tensão emocional).

Hemos preferido no contar con ellos por el momento y tratarlos junto con las unidades pluriverbales, en el futuro DiFraPE, en el que tenemos la esperanza de empezar a trabajar en un futuro próximo. En esta investigación nos tocará decidir la amplitud de nuestra consideración de las unidades fraseológicas, lo que implicará considerar (o no) las colocaciones como parte del universo fraseológico.

En esta presentación hemos intentado demostrar que un diccionario bilingüe contrastivo con función de diccionario de aprendi- 
zaje puede cubrir necesidades de uso que no cubre ningún diccionario monolingüe. Pero para cumplir con su función este tipo de diccionarios debe distinguirse bastante de los diccionarios bilingües tradicionales y de los de aprendizaje monolingües.

\section{Referencias}

DURÃO, Adja Balbino de Amorim Barbieri; SASTRE RUANO, María Ángeles; WERNER, Reinhold. "Lexicografia didáctica, dicionário bilíngue y lenguas afines: información semântica en el Diccionario Contrastivo Português-Español (DiCoPoEs)". In: NOMDEDEU RULL, Antoni; FORGAS, Esther; BARGALLÓ ESCRIVÁ, María. Tarragona: Publicaciones de la URV, 2012. p. 67-78.

Recebido em 15/12/2012

Aceito em 12/06/2013 